\title{
GMR
}

\section{Clinical efficacy of therapeutic intervention for subclinical hypothyroidism during pregnancy}

\author{
R. Ju' ${ }^{1}$ L. Lin ${ }^{2}$, Y. Long ${ }^{2}$, J. Zhang ${ }^{2}$ and J. Huang ${ }^{2}$ \\ ${ }^{1}$ Gynaecology and Obstetrics Department, Beijing Chuiyangliu Hospital, \\ Beijing, China \\ ${ }^{2}$ Obstetrics Department, Beijing Friendship Hospital, Capital Medical University, \\ Beijing, China \\ Corresponding author: L. Lin \\ E-mail: linli32145@sina.com
}

Genet. Mol. Res. 15 (4): gmr15049019

Received July 21, 2016

Accepted September 26, 2016

Published November 21, 2016

DOI http://dx.doi.org/10.4238/gmr15049019

Copyright $(2016$ The Authors. This is an open-access article distributed under the terms of the Creative Commons Attribution ShareAlike (CC BY-SA) 4.0 License.

ABSTRACT. This study explored the effects of levothyroxine (LT4) replacement therapy on pregnancy outcomes in patients with subclinical hypothyroidism $(\mathrm{SCH})$. We analyzed the effects on pregnancy outcomes with respect to gestational week when the desired thyroid-stimulating hormone (TSH) level was reached as well as the length of time required to reach the target level during L-T4 treatment. This study enrolled 457 patients diagnosed with SCH upon initial thyroid function screening. Subjects were assigned to the treatment group $(\mathrm{N}=184)$, and the control group $(\mathrm{N}=273)$. Two variables were analyzed in the treatment group: the gestational week when the target TSH level was achieved and the length of time required to reach the target level during treatment. Based on these criteria, the treatment group was further divided into subgroups, including three subgroups based on the time required to reach target levels ( $<4$ weeks, 4-8 weeks, and $>8$ weeks) and gestational week when the target TSH level was

Genetics and Molecular Research 15 (4): gmr15049019 
achieved (before the 12th, between the 12th-28th, and after the 28th gestational week). The overall risk of complications in the control group was significantly higher than in the treatment group $(\mathrm{P}<0.05)$. After L-T4 treatment, the incidences of premature rupture of fetal membranes (PROM), gestational diabetes mellitus, fetal macrosomia, and postpartum hemorrhage in the group with treatment duration $<4$ weeks were significantly lower than those in the groups with 4-8 and $>8$ weeks treatment duration $(\mathrm{P}<0.05)$. L-T4 treatment can significantly reduce the risks of adverse pregnancy outcomes in pregnant women with $\mathrm{SCH}$. The shorter the treatment duration required to reach the target TSH level and the earlier the gestational week when the target TSH level is achieved through treatment, the lower the incidence of complications.

Key words: Subclinical hypothyroidism; Levothyroxine; L-T4; Pregnancy outcomes

\section{INTRODUCTION}

The effects of subclinical hypothyroidism $(\mathrm{SCH})$ on pregnancy outcomes and whether SCH in pregnant women should be treated remain controversial. The "Guidelines for Diagnosis and Treatment of Thyroid Disease during Pregnancy and Postpartum", jointly developed by the Chinese Society of Endocrinology and the Chinese Society of Perinatal Medicine, state that " $\mathrm{SCH}$ during pregnancy increases the risks of adverse pregnancy outcomes as well as brain damage and neurodevelopmental sequelae in the offspring, but due to the lack of scientific evidence, this guideline neither opposes nor recommends L-T4 treatment for $\mathrm{SCH}$ in pregnant women with negative test to thyroid peroxidase antibodies (TPOAb)" (Chinese Society of Endocrinology and Chinese Society of Perinatal Medicine, 2012). The "Guidelines of the American Thyroid Association for the Diagnosis and Management of Thyroid Disease during Pregnancy and Postpartum" (2011) state that "SCH is related to adverse maternal and fetal outcomes. However, due to the lack of randomized controlled trials, there is insufficient evidence to clarify whether L-T4 treatment should be recommended for $\mathrm{SCH}$ women with negative thyroglobulin antibodies (TgAb) during pregnancy" (Stagnaro-Green et al., 2011). Given that both guidelines acknowledge that SCH during pregnancy can increase the incidence of adverse pregnancy outcomes, and studies have reported that early levothyroxine (L-T4) replacement therapy can prevent progression of SCH to CH (Helfand and Redfern, 1998) and significantly improve symptoms and signs. Given that SCH may cause adverse pregnancy outcomes for mothers and infants, based on examination of the pros and cons, most experts and guidelines recommend treatment.

In the present study, L-T4 was used as a therapeutic intervention and the enrolled $\mathrm{SCH}$ pregnant patients were fully informed. This study aimed to provide evidence for the clinical treatment of $\mathrm{SCH}$ in pregnant women by analyzing the effects of therapeutic interventions on pregnancy outcomes as well as differences in unexpected pregnancy outcomes according to durations required for serum TSH levels to achieve therapeutic standards with L-T4 treatment and the gestational weeks at which serum TSH levels reached the therapeutic standard during treatment.

Genetics and Molecular Research 15 (4): gmr15049019 


\section{MATERIAL AND METHODS}

\section{General information}

The subjects enrolled in this study included patients with $\mathrm{SCH}$ who gave birth and were registered in the maternity clinic at Beijing Friendship Hospital of Capital Medical University between October 2010 and September 2013. The inclusion criteria included: 1) patients without internal medicine and surgical chronic diseases such as chronic hypertension, diabetes mellitus, endocrine disorders, history of thromboembolism, severe gastrointestinal/ cardiopulmonary/liver diseases, or cancer before pregnancy; 2) patients without thyroid disease and autoimmune diseases before pregnancy (i.e., type 1 diabetes mellitus and systemic lupus erythematosus); 3) patients with live singleton confirmed by early B-ultrasound; 4) patients without a history of infertility or assisted reproduction; 5) patients not receiving treatment with thyroid hormones or antithyroid drugs; 6 ) patients with a median urine iodine concentration of $156.43 \pm 9.21 \mu \mathrm{g} / \mathrm{L}$ during early pregnancy; and 7) patients negative for TPOAb. A total of 457 qualified subjects who were screened, diagnosed, and confirmed to meet the inclusion criteria were divided into treatment $(\mathrm{N}=184)$ and control $(\mathrm{N}=273)$ groups.

The study protocol was approved by the Ethics Committees of Beijing Friendship Hospital of Capital Medical University, Beijing, and all participants provided written informed consent.

\section{Diagnostic criteria}

Using the 2.5th and 97.5th percentiles as the lower and upper limits of the normal values for thyroid stimulating hormone (TSH) and free thyroxin (FT4), a normal reference range of specific thyroid function during gestational weeks was established. Patients with TSH levels greater than the 97.5 th percentile and FT4 levels at or above the 2.5 th percentile and less than or equal to the 97.5 th percentile were diagnosed with $\mathrm{SCH}$.

\section{Treatment}

The treatment protocol adopted in this study was based on the recommendation in China "Guidelines for Diagnosis and Treatment of Thyroid Disease during Pregnancy and Postpartum" (2012): "III. SCH treatment and monitoring frequency in pregnancy are the same as in any other clinical hypothyroidism, for which the initial dose of L-T4 is determined based on the elevation of TSH level and the dose of L-T4 can be adjusted according to the TSH treatment goals". The treatment target concentration was set as a serum TSH level lower than the upper limit of the specific reference value during pregnancy (97.5th percentile). Subjects included in the study received treatment with oral L-T4 (Euthyrox, Merck Pharmaceuticals, $100 \mu \mathrm{g} /$ tablet) (Chinese Society of Endocrinology and Chinese Society of Perinatal Medicine, 2012).

\section{Study parameters}

Adverse pregnancy outcomes in the two groups of patients included premature rupture of fetal membranes (PROM), fetal macrosomia, gestational diabetes mellitus, hypertensive disorders in pregnancy, postpartum hemorrhage, preterm labor, oligohydramnios, fetal distress, and low birth weight (LBW).

Genetics and Molecular Research 15 (4): gmr15049019 


\section{Statistical methods}

IBM SPSS Statistics for Windows, version 19.0 was used for statistical analysis. The enumeration data are reported as percentages (\%) and intragroup comparisons were tested using $\chi^{2}$ tests. Measurement data are reported as means \pm standard deviations and intragroup comparisons were tested using $t$-tests. $\mathrm{P}<0.05$ indicated statistically significant differences.

\section{RESULTS}

\section{General study and control subjects characteristics}

General characteristics, including age, body mass index (BMI) at the first gynecological examination, body weight, exponential growth during pregnancy, baseline TSH level, and gestational week at the time of diagnosis, were compared. The differences in the general characteristics were not statistically significant $(P>0.05)$, indicating that the two groups were similar (Table 1).

Table 1. Intragroup comparison of general characteristics (means $\pm \mathrm{SD}$ ).

\begin{tabular}{l|c|c|c|c|c|c}
\hline Group & $\begin{array}{c}\text { Number } \\
\text { of cases }\end{array}$ & Age (years) & $\begin{array}{c}\text { Gestational week } \\
\text { at diagnosis }\end{array}$ & $\begin{array}{c}\text { Baseline TSH } \\
(\mu \mathrm{IU} / \mathrm{mL})\end{array}$ & $\begin{array}{c}\text { BMI at the first gynecological } \\
\text { examination }\left(\mathrm{kg} / \mathrm{m}^{2}\right)\end{array}$ & $\begin{array}{c}\text { Body weight exponential } \\
\text { growth }\left(\mathrm{kg} / \mathrm{m}^{2}\right)\end{array}$ \\
\hline Treatment & 184 & $29.31 \pm 3.36$ & $10.96 \pm 8.02$ & $5.09 \pm 1.58$ & $24.67 \pm 3.96$ & $3.77 \pm 2.65$ \\
\hline Control & 273 & $28.88 \pm 3.53$ & $10.21 \pm 9.27$ & $4.92 \pm 1.64$ & $24.23 \pm 3.61$ & $3.87 \pm 2.35$ \\
\hline P value & & 0.171 & 0.067 & 0.246 & 0.110 & 0.701 \\
\hline
\end{tabular}

Baseline TSH is the level at the time of the first gynecological examination (the time of enrollment) TSH, thyroidstimulating hormone; BMI, body mass index.

\section{Comparison of pregnancy outcomes between the two groups}

The overall risk of pregnancy complications in the control group was significantly higher than that in the treatment group [odds ratio $(\mathrm{OR})=1.219,95 \%$ confidence interval $(\mathrm{CI})=1.139-1.304]$, a significant difference $(\mathrm{P}<0.05)$. Intergroup comparison demonstrated that the risks for gestational diabetes mellitus $(\mathrm{OR}=1.938,95 \% \mathrm{CI}=1.267-2.964)$ and fetal macrosomia $(\mathrm{OR}=3.081,95 \% \mathrm{CI}=1.783-5.326)$ in the control group were significantly higher than those in the treatment group, and the differences were significant (both $\mathrm{P}<0.05$ ). The differences in the risks for PROM, pregnancy hypertension, preterm labor, oligohydramnios, postpartum hemorrhage, fetal distress, and LBW between the two groups were not statistically significant (all $\mathrm{P}>0.05$ ) (Table 2).

\section{Pregnancy outcomes in the study group and impact of the treatment duration required to achieve the target serum TSH levels}

Depending on the duration of treatment required to reach the target TSH level, the treatment group was divided into three subgroups $(<4$, between 4 and 8 , and $>8$ weeks). The intragroup differences in pregnancy outcomes between the three subgroups were analyzed using $\chi^{2}$ tests (Table 3). 
Table 2. Comparison of pregnancy outcomes between groups (n/\%).

\begin{tabular}{l|c|c|c|c|c}
\hline Pregnancy outcomes & Treatment group $(\mathrm{N}=184)$ & Control group (N=273) & P values & OR & $95 \%$ CI \\
\hline PROM & $28(15.2)$ & $57(20.9)$ & 0.127 & 1.372 & $0.909-2.071$ \\
\hline Gestational diabetes mellitus & $24(13.0)$ & $69(25.3)$ & $0.001^{\triangle}$ & 1.938 & $1.267-2.964$ \\
\hline Fetal macrosomia & $14(7.6)$ & $64(23.4)$ & $0.000^{\triangle}$ & 3.081 & $1.783-5.326$ \\
\hline Pregnancy hypertension & $11(6.0)$ & $21(7.7)$ & 0.481 & 1.287 & $0.636-2.604$ \\
\hline Preterm labor & $6(3.3)$ & $11(4.0)$ & 0.670 & 1.236 & $0.465-3.282$ \\
\hline Oligohydramnios & $9(4.9)$ & $9(3.3)$ & 0.390 & 0.674 & $0.273-1.666$ \\
\hline Postpartum hemorrhage & $7(3.8)$ & $9(3.3)$ & 0.772 & 0.867 & $0.329-2.286$ \\
\hline Fetal distress & $51(27.7)$ & $97(35.5)$ & 0.080 & 1.282 & $0.966-1.701$ \\
\hline LBW & $6(3.3)$ & $7(2.6)$ & 0.660 & 0.786 & $0.269-2.302$ \\
\hline Total & $105(57.1)$ & $252(92.3)$ & $0.000^{\triangle}$ & 1.219 & $1.139-1.304$ \\
\hline
\end{tabular}

OR, odds ratio; CI, confidence interval; PROM, premature rupture of fetal membranes; LBW, low birth weight. ${ }^{\mathrm{P}}$ $<0.05$.

Table 3. Treatment group: pregnancy outcomes according to the duration of treatment required to reach the target serum TSH level (N/\%).

\begin{tabular}{l|c|c|c|c|c|c}
\hline Pregnancy outcomes & $<4$ weeks $(\mathrm{N}=71)$ & $4-8$ weeks $(\mathrm{N}=69)$ & $>8$ weeks $(\mathrm{N}=44)$ & P1 value & P2 value & P3 value \\
\hline PROM & $4(5.6)$ & $12(17.4)$ & $12(27.3)$ & $0.029^{\triangle}$ & $0.001^{\triangle}$ & 0.210 \\
\hline Gestational diabetes mellitus & $3(4.2)$ & $10(14.5)$ & $11(25.0)$ & $0.036^{\triangle}$ & $0.001^{\triangle}$ & 0.161 \\
\hline Fetal macrosomia & $1(1.4)$ & $5(7.2)$ & $8(18.2)$ & $0.033^{\triangle}$ & $0.001^{\triangle}$ & 0.076 \\
\hline Pregnancy hypertension & $3(4.2)$ & $3(4.3)$ & $5(11.4)$ & 0.971 & 0.144 & 0.156 \\
\hline Preterm labor & $0(0)$ & $3(4.3)$ & $3(6.8)$ & 0.076 & $0.026^{\triangle}$ & 0.568 \\
\hline Oligohydramnios & $3(4.2)$ & $3(4.3)$ & $3(6.8)$ & 0.971 & 0.543 & 0.568 \\
\hline Postpartum hemorrhage & $0(0)$ & $4(6.3)$ & $3(6.8)$ & $0.040^{\triangle}$ & $0.026^{\triangle}$ & 0.826 \\
\hline Fetal distress & $20(28.2)$ & $24(34.8)$ & $19(43.2)$ & 0.399 & 0.098 & 0.370 \\
\hline LBW & $0(0)$ & $3(4.3)$ & $3(6.8)$ & 0.076 & $0.026^{\triangle}$ & 0.568 \\
\hline
\end{tabular}

P1: comparison between treatment times $<4$ and 4-8 weeks; P2: comparison between treatment times $<4$ and $>8$ weeks; P3: comparison between treatment times of 4-8 and $>8$ weeks. TSH, thyroid-stimulating hormone; PROM, premature rupture of fetal membranes; LBW, low birth weight. ${ }^{\circ}<0.05$.

The group with a treatment duration $<4$ weeks had a significantly lower incidence of PROM, gestational diabetes mellitus, fetal macrosomia, and postpartum hemorrhage compared to the group with treatment duration 4-8 weeks $(\mathrm{P}<0.05)$. The group with a treatment duration $<4$ weeks had a significantly lower incidence of PROM, gestational diabetes, fetal macrosomia, premature delivery, postpartum hemorrhage, and LBW infants compared to the group with treatment duration $>8$ weeks, a statistically significant difference $(\mathrm{P}<0.05)$.

\section{Pregnancy outcomes in the study group and the impact of the target TSH value according to gestational age}

The treatment group was also divided into three subgroups according to the gestational week when the target TSH level was reached after treatment: gestational age $<12$ weeks, 12 28 weeks, and $>28$ weeks. The difference in the incidence of complications between the three groups was measured using $\chi^{2}$ tests (Table 4 ).

The group with gestational age $<12$ weeks had a significantly lower incidence of PROM, gestational diabetes mellitus, and fetal macrosomia than did the 12-28-week gestation group, a statistically significant difference $(\mathrm{P}<0.05)$. The group with a gestational age of 12 weeks had a significantly lower incidence of PROM, fetal macrosomia, hypertensive disorders in pregnancy, and postpartum hemorrhage than did the $>28$-week gestation group, a statistically

Genetics and Molecular Research 15 (4): gmr15049019 
significant difference $(\mathrm{P}<0.05)$. The 12-28-week gestation group showed a significantly lower incidence of postpartum hemorrhage than did the $>28$-week gestation group $(\mathrm{P}<0.05)$.

\begin{tabular}{|c|c|c|c|c|c|c|}
\hline Pregnancy outcomes & $\begin{array}{c}<12 \text { weeks of gestation } \\
(\mathrm{N}=73)\end{array}$ & $\begin{array}{c}\text { 12-28 weeks of } \\
\text { gestation }(\mathrm{N}=96)\end{array}$ & $\begin{array}{c}>28 \text { weeks of gestation } \\
(\mathrm{N}=15)\end{array}$ & P1 value & $\mathrm{P} 2$ value & P3 value \\
\hline PROM & $5(6.8)$ & $18(18.8)$ & $5(33.3)$ & $0.025^{\triangle}$ & $0.003^{\triangle}$ & 0.195 \\
\hline Gestational diabetes mellitus & $4(5.5)$ & $20(20.8)$ & $0(0)$ & $0.005^{\Delta}$ & 0.353 & 0.051 \\
\hline Fetal macrosomia & $1(1.4)$ & $11(11.5)$ & $2(13.3)$ & $0.011^{\triangle}$ & $0.021^{\Delta}$ & 0.843 \\
\hline Pregnancy hypertension & $3(4.1)$ & $5(5.2)$ & $3(20)$ & 0.739 & $0.026^{\triangle}$ & 0.039 \\
\hline Preterm labor & $2(2.7)$ & $4(4.0)$ & $1(6.7)$ & 0.655 & 0.445 & 0.664 \\
\hline Oligohydramnios & $5(6.8)$ & $6(6.3)$ & $3(20)$ & 0.876 & 0.107 & 0.070 \\
\hline Postpartum hemorrhage & $1(1.4)$ & $5(5.2)$ & $3(20)$ & 0.182 & $0.002^{\triangle}$ & $0.039^{\triangle}$ \\
\hline Fetal distress & $25(34.2)$ & $31(32.3)$ & $7(46.7)$ & 0.789 & 0.362 & 0.275 \\
\hline LBW & $2(2.8)$ & $3(3.1)$ & $1(6.7)$ & 0.884 & 0.453 & 0.494 \\
\hline
\end{tabular}

P1: comparison between the group with gestational age $<12$ weeks and the group with gestational age 12-28 weeks; P2: comparison between the group with gestational age $<12$ weeks and the group with gestational age $>28$ weeks; P3: comparison between the group with gestational age 12-28 weeks and the group with gestational age $>28$ weeks. TSH, thyroid-stimulating hormone; PROM, premature rupture of fetal membranes; LBW, low birth weight. ${ }^{\mathrm{P}}<0.05$.

\section{DISCUSSION}

TPOAb is a serological marker of autoimmune thyroid disease that more commonly affects pregnant women. Hence, TPOAb is associated with adverse pregnancy outcomes (Lin et al., 2014). The impact of TPOAb-negative SCH on pregnancy outcomes and the necessity of medical treatment remain controversial, both at home and abroad. We addressed these controversies through a prospective cohort study. Our study group included TPOAb-negative pregnant women with SCH identified in the early pregnancy period during thyroid function screening. This study analyzed the necessity of medical treatment based on adverse pregnancy outcomes in the absence of L-T4 treatment, the gestational week when the desired TSH level was reached, and the duration of treatment required to reach the desired TSH level.

The effects of $\mathrm{SCH}$ on pregnancy and its outcomes at home and abroad are controversial. A previous study found that the pregnancy complications associated with SCH include gestational hypertension, preeclampsia, preterm labor, LBW, placental abruption, and postpartum hemorrhage (Helfand and Redfern, 1998). Another study reported that the incidence of gestational diabetes mellitus in pregnant women with SCH could reach $47 \%$ (Gallas et al., 2002). Casey et al. (2005) showed that the risk of adverse pregnancy outcomes in pregnant women treated for $\mathrm{SCH}$ was 2-3 times higher than that in pregnant women without treatment. Haddow et al. (1999) reported that pregnant women with SCH had decreased maternal-fetal thyroid hormone transportation, causing harm to fetal brain development (Hollowell et al., 1999). In a prospective multicenter fat-adapted-substrate oxidation in-trained-elite-runners study by Cleary-Goldman et al. (2008), thyroid function screening during early and midpregnancy stages of 10,990 pregnant women did not show a significant correlation between SCH and adverse pregnancy outcomes (Cleary-Goldman et al., 2008). Domestic studies have reported similar findings; Liu et al. (2009) found that the maternal and fetal outcomes of women with SCH during pregnancy showed no differences compared with the control group. The indicators for adverse pregnancy outcomes in this study were selected mainly based on previous research findings. For example, Helfand and Redfern (1998) reported that 
SCH-associated pregnancy complications include gestational hypertension, preeclampsia, preterm labor, LBW, placental abruption, and postpartum hemorrhage. Based on domestic and international guidelines, this study selected L-T4 as the first choice for thyroid hormone replacement therapy in pregnant women because the main role of the thyroid hormone is to increase metabolic activity and thus facilitate growth and development. Fetal development, especially brain development, depends on adequate maternal T4 levels, rather than T3 levels (Wartofsky, 2013). This study revealed that the overall risk of pregnancy complications in untreated SCH pregnant women was significantly higher than that in the L-T4 treatment group. Specifically, the risk of gestational diabetes mellitus was 1.938 times higher and the risk of fetal macrosomia was 3.081 times higher at the same time. Furthermore, the differences in the risks of PROM, hypertensive disorders in pregnancy, preterm labor, oligohydramnios, postpartum hemorrhage, fetal distress, and LBW between the treated and untreated groups were insignificant. These findings are consistent with those published by Negro et al. (2010) that administration of L-T4 to pregnant women with $\mathrm{SCH}$ can reduce the occurrence of pregnancy complications.

Fetal thyroid gland begins to secrete thyroid hormone from 12 weeks of gestation and the hypothalamic-pituitary-thyroid axis is functionally established at 18-20 weeks of gestation, indicating the maturity of thyroid function. Therefore, the thyroid hormone required by the fetal brain development before the 20th gestational week mainly comes from the mother's thyroid gland. Important brain developmental events that are closely related to thyroid hormones, such as neuron proliferation and migration in the brain, begin at the 12th gestational week (Rovet, 1999). Abalovich et al. (2007) retrospectively analyzed pregnancy outcomes in 114 pregnant women with hypothyroidism, including 52 cases of clinical hypothyroidism and 62 cases of SCH. They found that among the pregnant women with hypothyroidism who did not receive sufficient L-T4 therapy, $60 \%$ of patients with clinical hypothyroidism had miscarriage and $20 \%$ had preterm labor, while 71.4 and $7.2 \%$ of patients with SCH had miscarriage and premature labor, respectively. However, they discovered that among the pregnant women with hypothyroidism successfully treated with L-T4, 100\% of patients with clinical hypothyroidism and $90.5 \%$ of SCH patients had full-term labor, with a zero incidence rate of miscarriage. They concluded that the key factor deciding the impact of hypothyroidism on pregnancy is unrelated to the clinical or subclinical type, but rather whether timely and adequate treatment if offered; hence, hypothyroidism during pregnancy requires regular monitoring. Replacement therapy should be adjusted in a timely manner based on monitoring results. They also concluded that adverse pregnancy outcomes can be prevented through timely and effective treatment (Abalovich et al., 2007). Yu et al. (2010) believed that $\mathrm{SCH}$ diagnosed before the 8th gestational week could result in neuropsychological and motor function deficits in offspring and timely L-T4 treatment might restore the offspring's intelligence level to a level comparable to the offspring of a normal pregnancy. Thus, Yu et al. (2010) proposed that treatment for hypothyroidism should be initiated before the 12th week of gestation. Moreover, they stated that fetal brain development might still be impaired even if the hypothyroidism during pregnancy is mild and asymptomatic. Clinical studies have shown that an increasing need for thyroid hormone (or exogenous L-T4) begins within the first 4 to 6 weeks of pregnancy and is gradually enhanced from 16 to 20 weeks of pregnancy, ultimately reaching a steady state until delivery (Yu et al., 2010).

By studying the impact of the duration of treatment required to reach target TSH levels on pregnancy outcomes in the treatment group, the present study found that the incidences of

Genetics and Molecular Research 15 (4): gmr15049019 
PROM, gestational diabetes mellitus, fetal macrosomia, and postpartum hemorrhage in the group with a treatment duration $<4$ weeks were significantly lower than in the groups with treatment durations of 4-8 weeks and $>8$ weeks. In addition, the incidences of preterm labor and LBW were significantly decreased in the group with treatment duration $<4$ weeks compared to those in with treatment duration $>8$ weeks but did not show any significant differences compared with those in the group with a treatment duration of 4-8 weeks. The differences in the incidences of all complications were not significant between the group with treatment duration of 4-8 weeks and the group with treatment duration $>8$ weeks. These results suggest that the shorter the time required to reach the target serum TSH level after treatment, the fewer complications. In particular, in patients who achieved the target level within four weeks, treatment effectively reduced the incidence of pregnancy complications. In addition, this study investigated the impact of the gestational week when the target TSH level was reached on pregnancy outcomes. Our results demonstrated that the incidences of PROM, gestational diabetes mellitus, and fetal macrosomia in the group with gestational age $<12$ weeks significantly decreased compared to the groups with gestational ages of 12-28 weeks and $>28$ weeks. The incidences of gestational hypertension and postpartum hemorrhage in the group with gestational age $<12$ weeks were significantly lower than those in the group with gestational age $>28$ weeks, while the group with a gestational age of 12-28 weeks had a significantly reduced incidence of postpartum hemorrhage compared to the $>28$-week group. These results suggest that the earlier the gestational week when the target serum TSH level was reached in pregnant women with $\mathrm{SCH}$, the lower the incidence of complications. The incidence of pregnancy complications can be most effectively reduced when the desired level is reached before the 12th week of gestation. These findings are consistent with those published by Abalovich et al. (2007) and confirmed that timely and effective treatment of $\mathrm{SCH}$ can reduce the occurrence of adverse pregnancy outcomes.

The results of this study support the conclusions that L-T4 replacement therapy is necessary for treatment of SCH during pregnancy and that treatment should be timely and should reach treatment goals as quickly as possible in order to effectively reduce the incidence of adverse pregnancy outcomes. However, this study was limited by its small sample size; the conclusions require confirmation in large-scale prospective randomized controlled studies. Furthermore, the subjects included in this study did not undergo thyroid function evaluations before pregnancy, making it impossible to assess the impact of $\mathrm{SCH}$ illness duration on pregnancy outcomes and medication efficacy.

\section{Conflicts of interest}

The authors declare no conflict of interest.

\section{ACKNOWLEDGMENTS}

Research supported by the Capital Health Development Scientific Research Special Projects (\#2011-1002-04) and the Capital Health Development and Scientific Research Projects (\#2016-1-1113).

Genetics and Molecular Research 15 (4): gmr15049019 


\section{REFERENCES}

Abalovich M, Amino N, Barbour LA, Cobin RH, et al. (2007). Management of thyroid dysfunction during pregnancy and postpartum: an Endocrine Society Clinical Practice Guideline. J. Clin. Endocrinol. Metab. 92 (Suppl): S1-S47.http:// dx.doi.org/10.1210/jc.2007-0141

Casey BM, Dashe JS, Wells CE, McIntire DD, et al. (2005). Subclinical hypothyroidism and pregnancy outcomes. Obstet. Gynecol. 105: 239-245.http://dx.doi.org/10.1097/01.AOG.0000152345.99421.22

Cleary-Goldman J, Malone FD, Lambert-Messerlian G, Sullivan L, et al. (2008). Maternal thyroid hypofunction and pregnancy outcome. Obstet. Gynecol. 112: 85-92.http://dx.doi.org/10.1097/AOG.0b013e3181788dd7

Chinese Society of Endocrinology and Chinese Society of Perinatal Medicine (2012). Guidelines for the diagnosis and treatment of thyroid disease in pregnancy and postpartum. Chin. J. Endocrinol. Metabol. 28: 354-371.

Gallas PR, Stolk RP, Bakker K, Endert E, et al. (2002). Thyroid dysfunction during pregnancy and in the first postpartum year in women with diabetes mellitus type 1. Eur. J. Endocrinol. 147: 443-451._http://dx.doi.org/10.1530/ eje. 0.1470443

Haddow JE, Palomaki GE, Allan WC, Williams JR, et al. (1999). Maternal thyroid deficiency during pregnancy and subsequent neuropsychological development of the child. N. Engl. J. Med. 341: 549-555.

Helfand M and Redfern CC; American College of Physicians (1998). Clinical guideline, part 2. Screening for thyroid disease: an update. Ann. Intern. Med. 129: 144-158. http://dx.doi.org/10.7326/0003-4819-129-2-199807150-00020

Hollowell JG, Jr., Garbe PL and Miller DT (1999). Maternal thyroid deficiency during pregnancy and subsequent neuropsychological development of the child. N. Engl. J. Med. 341: 2016-2017.

Lin L, Zhang XL and Long Y (2014). Analysis of thyroid peroxidase antibody in early pregnancy. Genet. Mol. Res. 13: 5107-5114.http://dx.doi.org/10.4238/2014.July.7.3

Liu N, Bian XM, Gao YS, Qi H, et al. (2009). Clinical analysis of hypothyroidism or subclinical hypothyroidism during pregnancy. Chin. J. Perinatal Med. 12: 186-189.

Negro R, Schwartz A, Gismondi R, Tinelli A, et al. (2010). Universal screening versus case finding for detection and treatment of thyroid hormonal dysfunction during pregnancy. J. Clin. Endocrinol. Metab. 95: 1699-1707. http:// dx.doi.org/10.1210/jc.2009-2009

Rovet JF (1999). Congenital hypothyroidism: long-term outcome. Thyroid 9: 741-748. http://dx.doi.org/10.1089/ thy.1999.9.741

Stagnaro-Green A, Abalovich M, Alexander E, Azizi F, et al.; American Thyroid Association Taskforce on Thyroid Disease During Pregnancy and Postpartum (2011). Guidelines of the American Thyroid Association for the diagnosis and management of thyroid disease during pregnancy and postpartum. Thyroid 21: 1081-1125. http://dx.doi. org/10.1089/thy. 2011.0087

Wartofsky L (2013). Combination L-T3 and L-T4 therapy for hypothyroidism. Curr. Opin. Endocrinol. Diabetes Obes. 20: 460-466. http://dx.doi.org/10.1097/01.med.0000432611.03732.49

Yu XH, Wang WW, Teng WP, Shan ZY, et al. (2010). Levothyroxine treatment for subclinical hypothyroidism during pregnancy on the prospective study of the effect of offspring brain development. Chin. J. Endocrinol. Metab. 26: 921-925.

Genetics and Molecular Research 15 (4): gmr15049019 